\title{
Recent advances in the synthesis of monolithic metal-organic frameworks
}

\author{
Chongxiong Duan ${ }^{1,3}$, Yi Yu ${ }^{2}$, Jingjing $\mathrm{Li}^{1,3}$, Libo $\mathrm{Li}^{2}$, Bichun Huang ${ }^{4}$, Dongchu Chen ${ }^{1,3^{*}}$ and \\ Hongxia $\mathrm{Xi}^{2 *}$
}

\begin{abstract}
Although considerable achievements have been realized in recent years with respect to the syntheses of metalorganic frameworks (MOFs), majority of the developed MOFs are in the form of polydisperse microcrystalline powders, which cause dustiness, abrasion, and clogging and decrease pressure when used in industrial applications. Monolithic MOFs overcome these drawbacks and exhibit various promising characteristics. In this review, we present the recent advances associated with monolithic MOFs based on metal centers and a brief outline of the most prominent examples. Furthermore, the challenges and prospects associated with monolithic MOFs in terms of large-scale production and engineering applicability are analyzed based on our knowledge to conclude this review.
\end{abstract}

Keywords: metal-organic frameworks, monolithic structure, synthetic strategies, industrial practicability

\section{INTRODUCTION}

Metal-organic frameworks (MOFs), which are selfassembled via coordination bonds between the metal ions or clusters and organic ligands, are emerging as a class of fascinating porous organic-inorganic hybrid crystal materials [1,2]. They exhibit fascinating physicochemical characteristics, including ultrahigh specific surface area, abundant active sites, permanent porosity, and diverse topological structure $[3,4]$, making them potentially applicable in the fields of adsorption/separation, sensing, supercapacitors, drug delivery, functional peptidomics, and catalysis [5-11]. To date, majority of the reported MOFs are polydisperse microcrystalline powders. However, the utility of MOF powders in industrial applications is limited by various problems, including dustiness, abrasion, clogging, low packing densities, mass transfer limitations, and mechanical instability during the packing process [12-14]. For example, when packing HKUST-1 powder into an adsorption column for capturing $\mathrm{CH}_{4}$, the mass transfer resistance increases with increasing column time because of the obvious decrease in pressure [15]. Furthermore, the MOF powder as a catalyst exhibits disadvantages, such as difficulty in separation and recycling [16,17]. Although bulk architecture can be obtained via the simple padding of the MOF powder, the large amount of void space between the individual discrete crystallites in powders reduces the packing density $[18,19]$.

Compared with MOF powders, the densification of MOFs or shaping of MOFs into a monolithic state exhibits various advantages, including increased mechanical strength, packing density, and stability [20-22]. Since Küsgens et al. [23] initially reported a monolithic MOF $\left(\mathrm{Cu}_{3}(\mathrm{BTC})_{2}\right.$, BTC = benzene tricarboxylate $)$, their manufacturability and superiority have been given more attention compared with those of powder MOFs. To date, many advanced methods have been developed to synthesize monolithic MOFs, including direct synthesis from precursors [24], mechanical compression or extrusion [25], use of binders [26], sol-gel method [27], in situ combination with monolithic supports [28], and threedimensional (3D) printing technology [29]. Specific solvents and drying processes are usually required for the formation of the monolithic state when directly mixing the precursors [30]. Compression technology aims at adding the powdered MOF materials into the mold and subsequently applying a certain pressure to shape the materials into tablets, pellets, extrudates, or monoliths,

\footnotetext{
${ }^{1}$ School of Materials Science and Hydrogen Engineering, Foshan University, Foshan 528231, China

${ }^{2}$ School of Chemistry and Chemical Engineering, South China University of Technology, Guangzhou 510640, China

${ }^{3}$ Guangdong Key Laboratory for Hydrogen Energy Technologies, Foshan 528000, China

${ }^{4}$ School of Environment and Energy, South China University of Technology, Guangzhou 510006, China

* Corresponding authors (emails: Chendc@fosu.edu.cn (Chen D); cehxxi@scut.edu.cn (Xi H))
} 
according to the shape of the mold cavities [31]. Generally, to facilitate the shaping process and improve the density and mechanical strength of the final products, some binders can be considered [21]. In the sol-gel method, the precursors are first dispersed in a solvent to produce a low-viscosity solution, resulting in the formation of a stable sol system after a series of reactions, followed by the polymerization process to form monolithic gels with a 3D network [15]. Besides, in situ synthesis of MOF crystals on monolithic supporting materials provides a novel route to obtain monoliths. Combined with the popular 3D printing technology, MOFs can be transformed into agglutinable raw material and monolithic products can be constructed through layer-by-layer printing [32].

These methods can be used to prepare high-quality monolithic MOFs. Furthermore, they show considerable applicability to the improvement of industrial viability of powdered MOFs [21]. However, the major factor associated with the industrial application of monolithic MOFs is minimizing the loss of porosity when shaping and providing sufficient mechanical stability, which are necessary for engineering processes $[33,34]$. Therefore, advantages and disadvantages must be considered and shaping technologies must be optimized based on the inherent properties and practical applications of the specified MOFs. For example, the application of external pressure is a direct way to shape MOFs from powders. However, challenges such as structural collapse or amorphization remain even for some highly robust MOFs. In addition, the crystal topology was observed to exhibit anisotropic changes in MILs [35,36]. The addition of binders is another facile methodology to synthesize monolithic MOFs in any shape, with enhanced mechanical strength and thermal conductivity [37]. Pore blocking is another method that can be used to reduce the accessibility of channels to guests, resulting in challenges in finding the appropriate binder [38]. The sol-gel derivation of monolithic metal-organic gels (MOGs) is a promising route for shaping direct gelation from precursors and avoiding the microcrystalline powder state [39]. The key to the success of the sol-gel method lies in the formation of MOG structures from aggregated MOF particles (MOFPs), which requires prevention of the continuous growth of MOFPs [40,41]. This depends on the regulation of synthetic conditions that dominate crystallization and precipitation during gelation, including the temperature and concentrations of precursors or additives that disturb coordination [42-44]. The immobilization of MOF crystals on a porous support is an effective technique for obtaining hybrid features. Furthermore, extended applications can be obtained by optimizing the combination of physical and chemical performances [45]. Cordierite has become a preferred supporting monolith because of its low cost, low coefficient of thermal expansion, and improved mechanical properties [32]. In addition, carbon materials and silica monoliths are promising $[28,46]$. Some other techniques, such as paste extrusion [47] or the improved drying process [48], can be adopted based on the above methods to achieve excellent results, such as low cost, high porosity, high loading, and sufficient mechanical strength.

To date, several studies have summarized MOF shaping technologies suitable for industrial production and specific applications [20,49-53]. For example, Hou et al. [54] investigated the synthesis of gel-derived MOF monoliths and emphasized their importance in new functional materials. Nandasiri et al. [20] presented the densification strategies and applications of several representative MOFs in the form of pellets and emphasized the efforts in terms of mechanical properties. These studies are considerably important as reference to enhance the industrial practicability of monolithic MOFs. However, to the best of our knowledge, no special review has comprehensively summarized the selection and optimization of synthetic methods to fabricate MOF-based monolithic materials according to metal centers. Hence, this study will describe the state-of-the-art work related to the development of monolithic MOFs. Furthermore, this study focuses on the optimization of preparation methods for different types of MOFs. Finally, some problems that need to be solved in this field to bridge the gap between the production of MOFs and industrial demand are presented in this study.

\section{DIFFERENT TYPES OF MONOLITHIC MOFs BASED ON METAL IONS}

\section{Copper-based monolithic MOFs}

HKUST-1 (also known as $\mathrm{Cu}-\mathrm{BTC}$ or $\left[\mathrm{Cu}_{3}(\mathrm{BTC})_{2^{-}}\right.$ $\left.\left(\mathrm{H}_{2} \mathrm{O}\right)_{3}\right]$, BTC = benzene tricarboxylate $)$ is one of the most famous and best investigated rigid MOF models, wherein a binuclear copper complex is connected by carboxylate linkers in a paddle-wheel form to construct a cubic network [55]. The robust and porous structure provides attractive features, such as high specific surface area, well-defined channel properties, coordinatively unsaturated metal sites, and excellent thermal and chemical stabilities. Therefore, it has been widely selected to manufacture $\mathrm{Cu}$-based monoliths [56,57]. For example, Peterson et al. [58] pelletized HKUST-1 powders at a 
pressure of 10,000 psi and observed no impact on the crystal structure but a decrease in Brunauer-EmmettTeller (BET) surface area because of local structural collapse. Furthermore, Dhainaut et al. [59] reported a reduction in the textural properties of an MOF when compressed at $121 \mathrm{MPa}$. Therefore, the densification of HKUST-1 using high pressure is not commonly adopted.

In addition, Ahmed et al. [30] prepared a hierarchically porous $\mathrm{Cu}$-BTC monolith with micropores, mesopores, and macropores via a powder packing approach. Specifically, the precursor $\mathrm{Cu}\left(\mathrm{CH}_{3} \mathrm{COO}\right)_{2} \mathrm{H}_{2} \mathrm{O}$ and $\mathrm{H}_{3} \mathrm{BTC}$ powders were mixed and packed, followed by the addition of a mixture of ethanol and water, as shown in Fig. 1a. This was the first time that MOF monoliths have been directly obtained from precursors. The monoliths were formed via nanoparticle aggregation and did not contain a single crystalline phase. Furthermore, their Young's modulus was 10 times greater than that of the physically accumulated phase. Moreover, an ice-templating approach (control of the freezing and freezedrying processes) was developed to control the orientation of ice crystals during the directional freezing process with dimethylsulfoxide (DMSO) to form macropores and retain the inherent micropores (Fig. 1b) [48]. The samples obtained in both the aforementioned studies were shaped into a monolithic column with enhanced asymmetric mass transport and lower pressure drop during chromatographic separation.

A column-packed silica monolith with a homogeneous hierarchical network and sufficient mechanical properties was adopted to support $\mathrm{Cu}-\mathrm{BTC}$ nanoparticles by in situ combination [60]. The silica monoliths show unique advantages by which the inherent micro/meso/macropores form a penetrating network. The macroporous structures achieve enhanced mass transport efficiency. Based on the excellent catalytic performance of $\mathrm{Cu}$-BTC owing to the abundant unsaturated $\mathrm{Cu}(\mathrm{II})$ centers as Lewis acid active sites, the monolithic composite was used as an efficient catalyst to ensure continuous flow for the Friedländer reaction. Similarly, monolithic $\mathrm{Cu}-\mathrm{BTC} /$ silica on silica showed promise when developing a simple, low-cost, and efficient approach for the catalytic oxidation of alkylbenzene into corresponding ketones with a high yield and selectivity [46]. Ulker et al. [61] presented a sol-gel process to fabricate composite monoliths of silica aerogels with $\mathrm{Cu}$-BTC. This was realized by the dispersion, hydrolysis, condensation, gelation, aging, solvent exchange, and drying processes, among which the aging process played an essential role in improving the mechanical strength of gels via hydrolysis and condensation of the
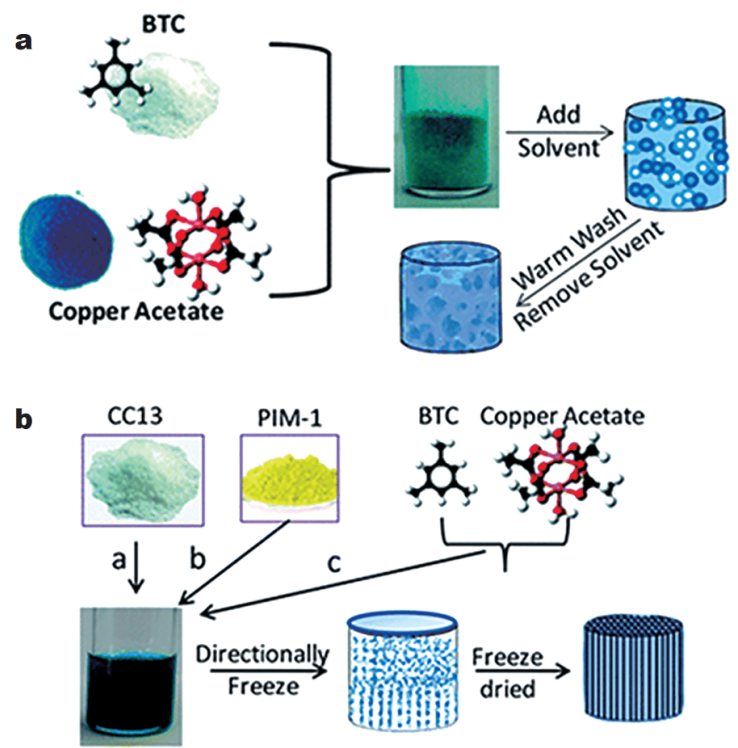

Figure 1 Schematic representations of the production of monoliths via (a) powder-packing synthesis, and (b) powder-packing synthesis coupled with ice-templating drying. Reprinted with permission from Refs [30,48]. Copyright 2014 \& 2015, Royal Society of Chemistry.

residual tetraethyl orthosilicate on gels. Furthermore, monoliths can be molded into any shape with varying porosities by simply changing the $\mathrm{Cu}$-BTC content to fit practical applications. In addition to the aforementioned catalytic capacity, the composite showed gas separation potential owing to the different accessibilities to the microporous and mesoporous domains and efficient gas storage, which were predicted via the grand canonical Monte Carlo simulations. Subsequently, graphene with enhanced electrical properties was selected to form a composite with $\mathrm{Cu}-\mathrm{BTC}$ via the nucleation, gelation, and drying processes [62]. The resulting monoliths showed high surface areas of $1078-1156 \mathrm{~m}^{2} \mathrm{~g}^{-1}$ and excellent electrical conductivities of $7.6 \times 10^{-6}$ to $6.4 \times 10^{-1} \mathrm{~S} \mathrm{~m}^{-1}$, which were proportional to the graphene content. Thus, the monolithic MOF composites have great potential in the field of electrochemical sensing.

The hierarchical porous carbon monoliths (HCMs) show great potential for capturing $\mathrm{CO}_{2}$ and a rapid regeneration ability, which can be attributed to the hierarchical network involving micrometer-sized macropores and micropores that can serve as gas diffusion and adsorption sites, respectively [64]. As a matrix, HCM with incorporated $\mathrm{Cu}-\mathrm{BTC}$ crystals allows further enhancement of the volumetric adsorption capacity of $\mathrm{CO}_{2}$. Furthermore, this was the first successful trial in which an MOF was synthesized within the macropores in HCM 
[63]. HCM was cured by a gel in any shape in which $\mathrm{Cu}$ BTC was formed via in situ synthesis. This was followed by the step-by-step impregnation and crystallization procedures to form $\mathrm{HCM}-\mathrm{Cu}_{3}(\mathrm{BTC})_{2}$ composites (Fig. 2). The monolithic composite exhibited an enhanced $\mathrm{CO}_{2}$ uptake of $22.7 \mathrm{~cm}^{3} \mathrm{~cm}^{-3}$, more than the initial $\mathrm{HCM}$ $\left(12.9 \mathrm{~cm}^{3} \mathrm{~cm}^{-3}\right)$ under identical conditions. This study created a new composite method to load an MOF for practical application in the field of $\mathrm{CO}_{2}$ capture.

The extrusion of 3D printing technology or direct ink writing is a flexible, low-waste, and low-cost processing method that retains the structural integrity and performance [66]. Gels containing only Cu-BTC nanoparticles and ethanol exhibit ideal rheological characteristics; therefore, they can be used as a medium for 3D-printed (3DP) MOF monoliths (Fig. 3). In addition, other $\mathrm{Cu}-$ based monolithic MOFs have been reported in recent years. For example, $\mathrm{Cu}(\mathrm{II})$ hydroxide monolith was fabricated using $\mathrm{CuCl}_{2}$ and propylene oxide via a sol-gel process with phase separation. This was followed by coordination replication into a $\mathrm{Cu}$-BTC monolith exhibiting high crystallinity, a surface area of $1315 \mathrm{~m}^{2} \mathrm{~g}^{-1}$, and sufficient mechanical properties in the presence of $\mathrm{H}_{3} \mathrm{BTC}$ [65]. This study confirmed the possibility that hydroxide materials can be developed into pure MOFs in terms of monoliths.

In addition, monolithic MOFs in the form of thin films, i.e., surface-mounted MOFs (SURMOFs) have been widely developed over the past years [67]. Compared with common monolithic MOFs, SURMOFs have high mass transfer rates, ultrathin thickness, and high surface-tovolume atom ratios $[68,69]$. Owing to these characteristics, SURMOFs have enormous potential of integrating multifunctional MOF thin films into miniaturized optoelectronics, sensors, lab-on-a-chip, and microfluidic devices [70,71]. For example, Gu et al. [72] reported the synthesis of HKUST-1 SURMOFs in a layer-by-layer fashion using liquid-phase epitaxy by employing a dipping robot. The as-synthesized highly crystalline HKUST1 SURMOFs enable the band gap in HKUST-1 to be experimentally determined using ellipsometry. Subsequently, the same group reported the anisotropic thermal expansion behavior of HKUST-1 SURMOFs [73]. Usually, these investigations are difficult to conduct for MOF powders and common monolithic MOFs.

\section{Iron-based monolithic MOFs}

To date, the research on iron-based monolithic MOFs has mainly focused on MIL-100(Fe), which comprises iron trimeric octahedral clusters and BTC linkers [74]. MIL-

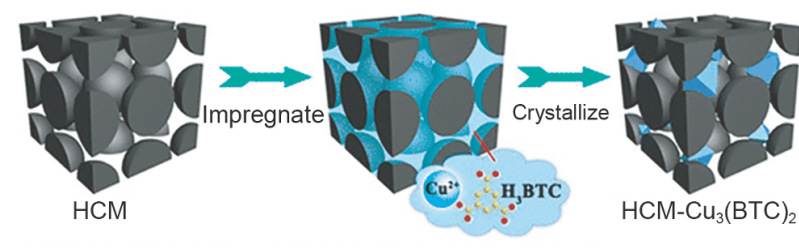

Figure 2 Schematic diagram of the fabrication of $\mathrm{HCM}-\mathrm{Cu}_{3}(\mathrm{BTC})_{2}$. Reprinted with permission from Ref [63]. Copyright 2012, American Chemical Society.

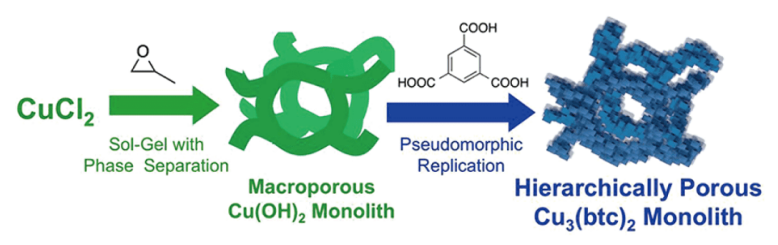

Figure 3 Schematic diagram of the 3DP-HKUST-1. Reprinted with permission from Ref [65]. Copyright 2015, Royal Society of Chemistry.

$100(\mathrm{Fe})$ contains three types of pores with diameters of 8.7, 25 , and $29 \AA$ to obtain a zeolite structure, allowing accessibility of guest molecules and ensuring adherence with the design of adsorption or catalytic materials [75]. Lohe et al. [76] presented the first example of a monolithic MOF aerogel (Fe-BTC) with high micro- and macroporosity. Compared with the powdered product, which was aged for $20 \mathrm{~h}$ before Soxhlet extraction and air-dried overnight at $80^{\circ} \mathrm{C}$, the as-synthesized Fe-BTC MOF monolith exhibited a considerably higher total pore volume of $5.62 \mathrm{~cm}^{3} \mathrm{~g}^{-1}$ at a relative pressure $\left(P / P_{0}\right)$ of 0.99 . The strategy of developing a high-porosity MOF monolith with any shape and size resulted in the development of a new concept for the design and application of MOFs as catalysts or catalyst supports; however, the low density and amorphous structure are issues that must be addressed.

Wickenheisser et al. [77] proposed a novel polymerization method to embed the presynthesized MOFs into a macroporous oil-water high internal phase emulsion (HIPE) foam based on $\mathrm{N}$-isopropyl acrylamide (NIPAM). As shown in Fig. 4, the as-synthesized MIL100(Fe)@NIPAM exhibited a monolith. Furthermore, the scanning electron microscopy (SEM) image indicates that the monolithic MIL-100(Fe)@NIPAM material exhibited obvious microporous structures. The average size of the MIL particles attached to the HIPE surface was 2-5 $\mu \mathrm{m}$. In addition, prepolymerization of the HIPE emulsion before the addition of MOF powders was an indispensable procedure to obtain MIL@NIPAM monoliths; 


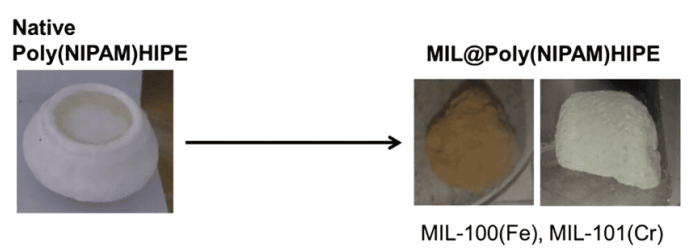

Figure 4 Schematic diagram of the preparation of MIL@NIPAM monoliths $(\mathrm{MIL}=\mathrm{MIL}-100(\mathrm{Fe}$ or $\mathrm{Cr}))$. Reprinted with permission from Ref [77]. Copyright 2016, Elsevier.

otherwise, severe channel blocking would occur. However, the pore blocking effect could be observed to some extent when using NIPAM and $N, N^{\prime}$-methylenebisacrylamide (MBA), resulting in a drastic reduction of the specific surface area from the expected values of 800 (containing $37 \mathrm{wt} \% \mathrm{MIL}$ ) and $1650 \mathrm{~m}^{2} \mathrm{~g}^{-1}$ (containing 78 $\mathrm{wt} \% \mathrm{MIL}$ ) to the measured values of 230 and $300 \mathrm{~m}^{2} \mathrm{~g}^{-1}$, respectively; however, these values were slightly greater than those of the native HIPE material $\left(20 \mathrm{~m}^{2} \mathrm{~g}^{-1}\right)$. Consequently, the composite showed a slightly enhanced water uptake capacity when compared with HIPE. Fortunately, the pore blocking effect was resolved by Wang et al. [78] by adding a small amount of poly(vinyl alcohol) (PVA) as the stabilizer when preparing monolithic MOFs.

In addition, MIL-100(Fe) can be used as a precursor for the synthesis of metal-organic xerogel (MOX) composite monoliths. For example, Mahmood et al. [79] constructed a highly active Fe-based nanoporous carbon (NPC) carbonized from MOXs as a positive electrode for asymmetric supercapacitors (Fig. 5). Here MOX monoliths were obtained via a facile solvothermal process, which resulted in high surface areas, very low densities, and hierarchical channels. The $\mathrm{Fe}_{3} \mathrm{O}_{4}$ active sites were preserved at an appropriate carbonization temperature, and the electrode exhibited an outstanding capacity retention of $500 \mathrm{~F} \mathrm{~g}^{-1}$ for 5000 charge-discharge cycles at a high current density of $8 \mathrm{~A} \mathrm{~g}^{-1}$.

\section{Zinc-based monolithic MOFs}

Zeolitic imidazolate frameworks (ZIFs), such as ZIF-4 and ZIF-8, are representative materials of zinc-based MOFs exhibiting highly tunable porosity, excellent thermal stability, and moisture resistance [80,81]. However, their utility in many applications is restricted by the inability to process the microcrystalline powders produced during their syntheses. Therefore, many advanced methods have been developed to prepare monolithic ZIF materials. For example, Tian et al. [24] reported the manufacturing of a transparent and robust ZIF-8 monolithic material via a facile and straightforward technique that did not involve the usage of binders or high pressure. The as-synthesized ZIF-8 monolith maintained the channel properties of powder crystals and exhibited a high pore volume. The key to the success is attributed to the presence of ZIF- 8 precursors and the mild and slow drying process performed with respect to the products. Researchers have focused on analyzing the mechanical properties of the monolith, thereby indicating that the elastic modulus and hardness of the material were considerably higher than those of the ZIF-8 single crystals and even those of previous monoliths. Furthermore, this method was successfully used to develop another ZIF-based material (ZIF-4); however, no further studies were conducted with respect to the mechanics of this method.

Widmer et al. [83] established another process based on the field-assisted sintering technique (FAST) to study the thermal and mechanical properties of the ZIF-4 monolith. This investigation was conducted for the very first time in the field of MOFs. Compared with the direct sintering method, FAST combines two steps, including powder compaction and heat treatment, into one step [82]. The machine rams the uniaxially compressed sample, and the electrodes simultaneously generate a direct current pulse through punches and graphite molds, producing thermal

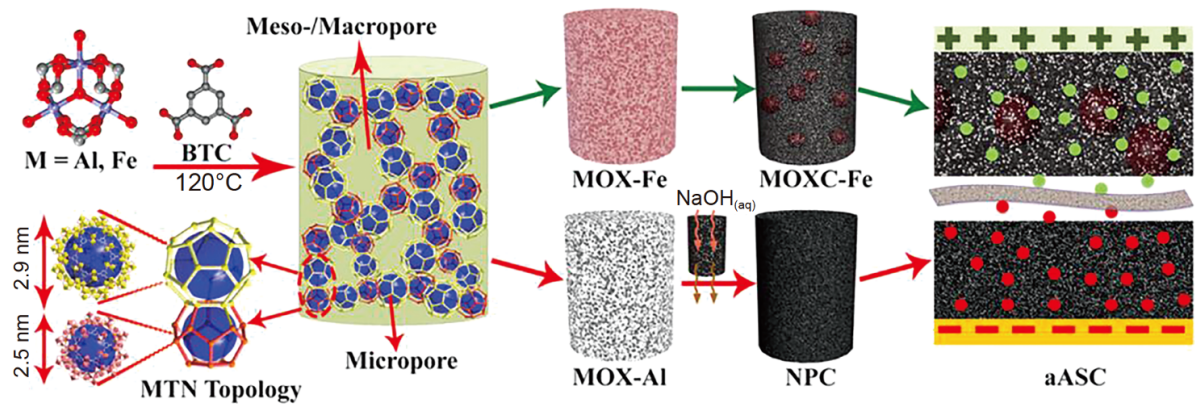

Figure 5 Schematic diagram of the preparation of MOXs composite monoliths derived from MIL-100(Fe). Reprinted with permission from Ref [79]. Copyright 2016, American Chemical Society. 
resistance to fuse the particles (Fig. 6). Compared with the direct mechanical densification reported by Zacharia et al. [35], the application of hot pressure allowed the monolith to avoid the formation of an amorphous structure, thereby maintaining the internal microporous properties. The material exhibited low elastic moduli and hardness values, which can be attributed to the response of the soft grain support and uniaxial compression during the sintering process. However, controlling the sintering conditions to regulate the mechanical and structural properties of the materials remains one of the future research directions.

In addition to the pure monolithic ZIF materials, some monolithic ZIF composites have been synthesized recently. For example, Mehta et al. [84] successfully used $\mathrm{SnO}_{2}$ nanoparticles (NPs) in situ within a monolithic ZIF8 sample through the sol-gel process coupled with an in situ combination at room temperature for $10 \mathrm{~min}$. This strategy does not require complicated synthesis conditions (e.g., binders, additives, or high pressures) and avoids structural amorphization as well as pore collapse. The resulting $\mathrm{SnO}_{2} @_{\text {mono }} \mathrm{ZIF-8}$ exhibited the effective photocatalytic activity of NPs and the size repulsion of micropores in ZIF-8 particles, which allowed only water and $\mathrm{H}_{2} \mathrm{O}_{2}$ to contact the active sites during the photodegradation reaction. Furthermore, reusability facilitates the promising application of the ZIF- 8 monolith in the field of sewage treatment.

\section{Aluminum-based monolithic MOFs}

Recently, two novel nanoporous monolithic materials, i.e., MIL-100(Al) xerogel and aerogel, with hierarchically porous structures have been reported for the high-efficiency removal of microcystin-LR (MC-LR) through economical solvothermal synthesis (Fig. 7) [85]. The main difference between the synthesis methods of MIL-

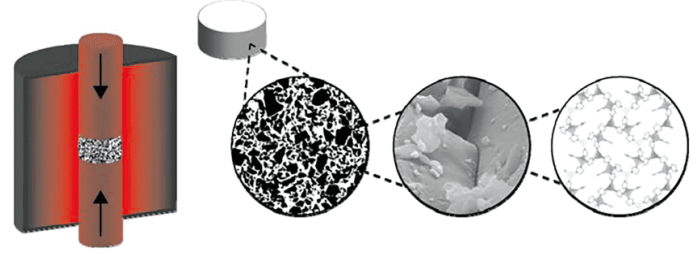

Figure 6 Schematic diagram of the fabrication of ZIF-4 monolith using a pressure-assisted sintering technique. Reprinted with permission from Ref [83]. Copyright 2018, American Chemical Society.

$100(\mathrm{Al})$ xerogel and aerogel is in the drying process; specifically, the former was slowly dried at ambient temperature, whereas the latter was obtained using a supercritical $\mathrm{CO}_{2}$ drying process, causing some differences in pore characteristics. The $\mathrm{N}_{2}$ adsorptiondesorption tests showed that the xerogel sample exhibited good surface chemistry with sufficient metal sites and carboxyl groups, resulting in an excellent adsorption capacity of $9007 \mu \mathrm{g} \mathrm{g}^{-1}$ at the initial MC-LR concentration of $10,000 \mathrm{ppb}$. The remaining concentration of MC-LR can be as low as $0.093 \mu \mathrm{g} \mathrm{L}^{-1}$, which is considerably lower than that of the standard concentration of drinking water.

In addition, cage-type MOF crystals with mesopores or corresponding MOF polymers as monolithic columns have been widely studied in the field of solid-phase microextraction (SPME) [86]. Specifically, the Al-based MOF monolith exhibits good extraction ability, which can be attributed to its mesoporous channels and unique breathing effect. Recently, Li et al. [87] assembled hierarchically porous Al-MOA (MOA = metal-organic aerogel) by introducing mesospaces via a versatile and facile template-assisted technique based on the two-step gelation of nanosized MOF particles (nMOFPs) containing well-defined microporous structures (Fig. 8). The result-
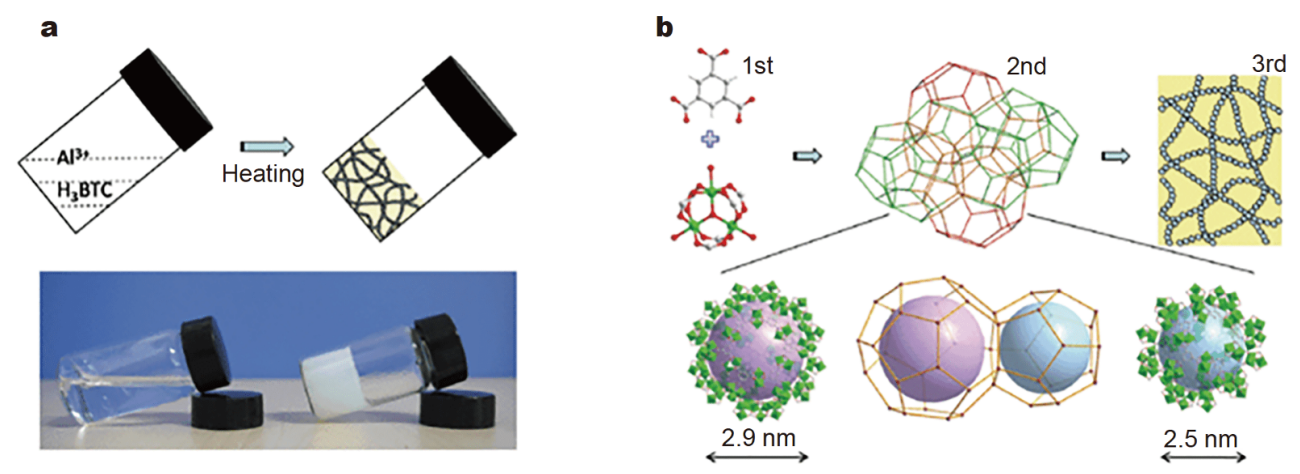

Figure 7 Schematic illustration of (a) transformation from solution to MIL-100(Al) monolith and (b) structure of MIL-100(Al) monolith. Reprinted with permission from Ref [85]. Copyright 2012, Royal Society of Chemistry. 


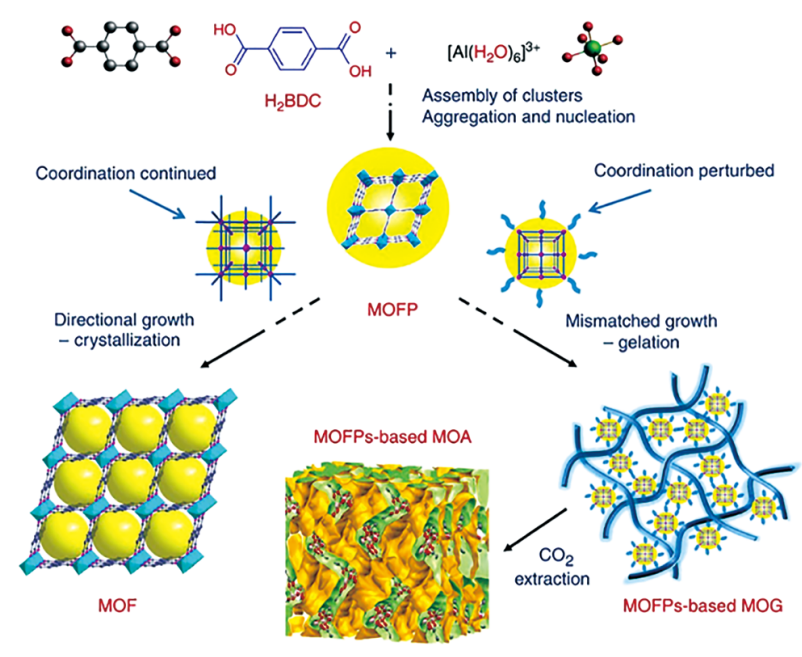

Figure 8 Schematic representation of the formation of initial MIL-53 (Al), and the resultant MOFP, MOG and MOA. Reprinted with permission from Ref [87]. Copyright 2013, Macmillan Publishers Limited.

ing Al-MOA exhibited the advantages of both porous MOFs and aerogels such as high specific surface area, tunable porosity, convenient chemical modification, and low density. The $\mathrm{H}_{2} / \mathrm{CO}_{2}$ or dye (congo red and brilliant blue R-250) molecules in MOA monolith exhibited excellent accessibility. In addition, the aerogel can be applied to SPME fiber coating with high molecular diffusion, outstanding extraction, and enriched performance, allowing it to compete with other materials having high capacity, selectivity, and sensitivity [88].

Similarly, Lirio et al. [89] prepared Al-based MOFPs, including MIL-53, MIL-68, CYCU-4, and DUT-5, in an aqueous phase. This was followed by the formation of monolithic composite columns using butyl methacrylateco-ethylene dimethacrylate (BMA-EDMA) using an ionic liquid $\left(\left[\mathrm{C}_{6} \mathrm{mim}\right]\left[\mathrm{BF}_{4}\right]\right)$ coupled with the microwave-assisted polymerization procedure for SPME of penicillin in river water and milk samples. The optimum extraction recovery of all the Al-based monolithic compounds toward penicillin was tested and evaluated; among the tested compounds, the MIL-53(Al)-polymer exhibited superior extraction recovery (river water: $80.8 \%-90.9 \%$; milk: $81.1 \%-100.7 \%$ ), low relative standard deviation (river water: <6.7\%; milk: $<7.1 \%$ ), low detection limit $\left(0.06-0.26 \mu \mathrm{g} \mathrm{L}^{-1}\right)$, and reusability, which can be attributed to the strong electrostatic interaction between the analyte and adsorbent and the unique breathing effect of MIL-53(Al). The simple synthetic process and high adsorption performance provide a good reference for other materials for application as adsorbents in SPME. This strategy can also be applied to fabricate other monolithic
MOFs (e.g., MIL-101(Cr)) with low cost, good recovery percentage, and high reusability [90].

Hastürk et al. [91] reported a novel shaping strategy, i.e., phase separation technique with PVA, to construct Alfum@PVA (Alfum = aluminum fumarate) and MIL101(Cr) monoliths. The pore volume of Alfum@PVA was greater than that expected on the basis of MOF loading, which can be attributed to the additional mesopores formed at the MOF-polymer interface, resulting in an increase in water uptake when compared with that in pure MOFs. However, this phenomenon was not observed in MIL-101(Cr) monoliths, resulting in decreased water loading (approximately $80 \%$ of that observed in pure MOFs). In addition, among all the prepared monoliths, the mechanical resistance was the highest when the Alfum loading was $65 \mathrm{wt} \%$ and decreased with the increasing loading capacity. Furthermore, the investigation of three different drying processes, including vacuum drying, supercritical drying, and freeze-drying, indicated that vacuum drying was the optimal choice to maintain the monolith and avoid shrinkage. However, freeze-drying is advantageous because ice-templated macroporous monoliths can be obtained after the removal of ice crystals generated during the freezing process; this approach was successfully applied to shape the HKUST-1 monolith $[48,92]$. Based on this principle, a research group adopted freeze-drying (also known as the freeze-casting method) coupled with the phase separation technology for polymers to prepare Alfum, MIL-160(Al), and MIL-101(Cr) monolithic composites [93]. Six hydrophilic polymer binders, including polyacrylic acid (PAA), sodium polyacrylate (PAANa), polyethylene glycol (PEG), polyethylene imine (PEI), PVA with two different hydrolysis degrees, 88 and 98, and polyvinyl pyrrolidone (PVP), were used. The $\mathrm{N}_{2}$ sorption tests showed that MIL-160(Al) with PVA(98) exhibited the best porosity corresponding to the MOF mass fraction, whereas the material containing PEI ranked low because of the strong interaction of PEI with MOF, resulting in a nonnegligible pore blocking effect. In addition, MIL-101 (Cr) with PEI showed heavy pore blocking. Furthermore, in terms of water loading, the monolithic MIL-160(Al) 80@PAA, PVA(98), PVA(88)4, and PVP exhibited highly satisfactory results with approximately $100 \%$ of the calculated water loading when $P / P_{0}=0.20$. However, the Alfum composites exhibited a varying water uptake of $87 \%-114 \%$ when $P / P_{0}=0.35$. The facile operation and satisfactory results obtained with respect to water loading enhance the reference value of this study when preparing other monolithic MOF composites. 


\section{Zirconium-based monolithic MOFs}

UiO-66 $\left(\left[\mathrm{Zr}_{6} \mathrm{O}_{4}(\mathrm{OH})_{4}(\mathrm{BDC})_{6}\right], \mathrm{BDC}=1,4\right.$-benzenedicarboxylate) and $\mathrm{UiO}-66-\mathrm{NH}_{2}$ are the most interesting MOFs in the family of $\mathrm{Zr}$-based MOFs because of their high thermal, mechanical, and chemical stabilities, high porosity, and tunable structural properties [94]. Thus, UiO-66 and its derivates are the most studied $\mathrm{Zr}$-based MOFs, whereas other Zr-based MOFs, such as UiO-67, MOF-801, and NU-1000, have rarely been investigated. For example, Dhainaut et al. [59] presented a general methodology based on quantitative descriptors for the densification of pure $\mathrm{UiO}-66, \mathrm{UiO}-66-\mathrm{NH}_{2}$, and $\mathrm{UiO}-67$ using a tableting facility. Furthermore, the impact of external pressure on the porous features, mechanical strength, and bulk density was measured. The results indicate that the bulk density of the tablet can be increased by 1.8-3.4 times and that the specific surface area (SSA) decreased by $0-30 \%$ compared with those of the initial MOF powders obtained via compression. The aforementioned researchers have reported the proportional relation between mechanical stability and bulk density for the very first time. Only UiO-66 and UiO-66$\mathrm{NH}_{2}$ were confirmed to exhibit long-term stability in the presence of water vapor. This study has considerable reference value for the densification of other materials according to their applications. The mechanical strengths of the millimeter-scale UiO-66 and UiO-66- $\mathrm{NH}_{2}$ fabricated via the wet granulation strategy using rho-alumina as the binder were studied and exhibited average crushing strengths of 4.7 and $2.5 \mathrm{~N}$, respectively.

Based on the excellent tolerance of high pressure, Bambalaza et al. [95] confirmed that UiO-66 can retain at least $95 \%$ of the initial porosity and that it shows an enhanced total volumetric hydrogen storage capacity under high external pressure (665 $\mathrm{MPa}$ or $97,000 \mathrm{psi}$ ), which is unprecedented for the compaction of MOFs. However, the UiO-66 powder sample was synthesized using a conventional method followed by compaction at applied pressures of 150, 290, 440,590, and $665 \mathrm{MPa}$ in a Specac Manual Hydraulic Press for $15 \mathrm{~min}$. The results obtained when using the highest pressure demonstrated the retention of crystallinity and the enhancement of packing density from 0.57 to $1.45 \mathrm{~g} \mathrm{~cm}^{-3}$. When the packing density was increased, the $\mathrm{H}_{2}$ uptake of the monolith reached an unprecedented value of $74 \mathrm{~g} \mathrm{~L}^{-1}$ at $77 \mathrm{~K}$ and 100 bar $\left(13 \mathrm{~g} \mathrm{~L}^{-1}\right.$ at $\left.298 \mathrm{~K}\right)$ compared with the value of $29 \mathrm{~g} \mathrm{~L}^{-1}\left(6 \mathrm{~g} \mathrm{~L}^{-1}\right.$ at $\left.298 \mathrm{~K}\right)$ obtained for the powder sample. The study offers promise with respect to the $\mathrm{H}_{2}$ storage targets set by the United States Department of Energy.
Connolly et al. [96] prepared high-density and centimeter-scale monoliths of UiO-66 from gels without using binders or applying high pressure. This allowed varying the hierarchical system and modulating the shape of monoliths depending on the washing and drying procedures (Fig. 9). The adsorption of $\mathrm{CH}_{4}$ and $\mathrm{CO}_{2}$ in the synthesized monoliths indicated a promising Type-II isotherm, which contributed to a higher volumetric uptake when compared with that of the microporous UiO66 powder with a Type-I curve. Similarly, Vilela et al. [97] reported the production of hierarchically porous and centimeter-scale UiO-66- $\mathrm{NH}_{2}$ monolithic single pieces with a controlled shape and mechanical robustness from ethanolic gels. The hierarchical structure of monolithic UiO-66- $\mathrm{NH}_{2}$ was studied. $\mathrm{N}_{2}$ sorption proved the existence of abundant micro- and mesopores; mercury intrusion porosimetry further proved the presence of macropores, fractures, and even intergranular pores and confirmed that less concentrated gels increased the porosity. Focused ion beam scanning electron microscopy (FIB-SEM) is a powerful mechanism to reconstruct the 3D models of porous UiOs. The strategy of synthesizing monolithic MOF from gelated samples via a specific drying process was successfully applied to other Zr-based MOFs such as MOF-801, MOF-808 and NU-1000 [98]. Moreover, the gelated UiO-66 sample was molded into monolithic spheres with a diameter of $600 \mathrm{~mm}$ using the oil drop method. The creative design can be applied in packed-bed catalytic or adsorptive applications, wherein the mass transfer constraints can be reduced using a hierarchical system.

\section{Chromium-based monolithic MOFs}

MIL-101(Cr) is a chromium(III) terephthalate MOF with micro-/mesoporous structures and high Langmuir surface areas of up to $5900 \pm 300 \mathrm{~m}^{2} \mathrm{~g}^{-1}$. This compound is a cage-type material with diameters of 3 and $29 \AA$, and can provide abundant channels and active sites for gas adsorption or other applications. Furthermore, it can avoid pore blockage when using polymers or binders to some extent $[77,99]$. In addition, the excellent stability of MIL$101(\mathrm{Cr})$ allows maintaining the structural integrity in air for several months, which is promising for the syntheses and applications of monolithic MOFs. $\mathrm{Cr}$ (III) gels with a rigid bridging carboxyl group, such as BDC, BTC or 1,4naphthalenedicarboxylate (NDC), can be obtained only at high temperatures (e.g., $80^{\circ} \mathrm{C}$ ), which can be attributed to the slow substitution kinetics associated with aqueous $\mathrm{Cr}$ (III) [100]. The high-temperature effect causes the thermal irreversibility of monolithic materials, which exhibit 

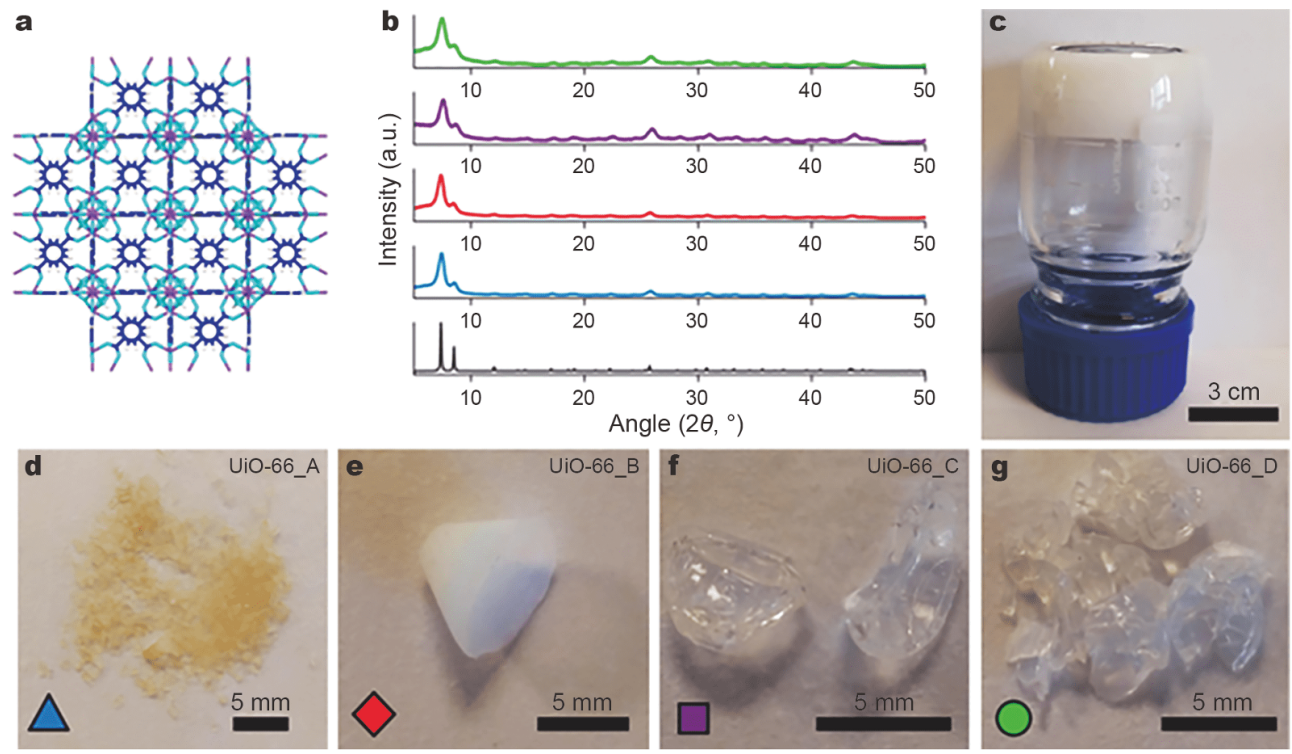

Figure 9 (a) Graphical representation of the crystal structure of UiO-66; (b) XRD patterns of UiO-66 samples; (c) UiO-66 gel for synthesis of monoliths; and (d-g) the optical images of UiO-66 monoliths: UiO-66_A (UiO-66 gel washed in ethanol and dried at 200 $\left.{ }^{\circ} \mathrm{C}\right)$; UiO-66_B (UiO-66 gel washed in ethanol and dried at $30^{\circ} \mathrm{C}$ ); UiO-66_C (UiO-66 gel washed in DMF and dried at $30^{\circ} \mathrm{C}$ ); UiO-66_D (UiO-66 gel washed in DMF with extended centrifugation and then dried at $30^{\circ} \mathrm{C}$ ). Reprinted with permission from Ref [96].

good application prospects at high temperatures.

Recently, Hong et al. [47] prepared the MIL-101(Cr) monolith from as-synthesized powders using bentonite clay as the binding agent via the paste extrusion technique (Fig. 10a). This method is economical and energysaving, and the monolithic product showed reusability, good porosity, and strong mechanical resistance. Furthermore, the mass transfer properties (e.g., equilibrium adsorption capacity, stoichiometric and equilibrium time, and mass transfer zone velocity) and the length of the samples were evaluated based on the $\mathrm{CO}_{2}$ adsorption breakthrough curves. For example, the equilibrium adsorption capacity for $40 \%(v / v) \mathrm{CO}_{2}$ on the MIL-100(Cr) monolith was $1.95 \mathrm{mmol} \mathrm{g}^{-1}$ at 2 bar, which is approximately $38 \%$ greater than those of the corresponding powders.

Wickenheisser et al. [101] adopted 2-hydroxyethylmethacrylate (HEMA) as a hydrophilic monomer to synthesize the poly(HIPE) material and embedded MIL101(Cr) into it to fabricate monolithic MIL-101@HIPE composites (Fig. 10b). The maximum loading of MIL was up to $59 \%$; however, the BET specific surface area of the composites was less than that of the corresponding loading. This result can be attributed to the partial pore blocking, which causes the reduction of maximum exchange toward methanol $\left(0.37 \mathrm{~g} \mathrm{~g}^{-1}\right)$ and water vapor $\left(0.29 \mathrm{~g} \mathrm{~g}^{-1}\right)$ when compared with those of pure MOFs
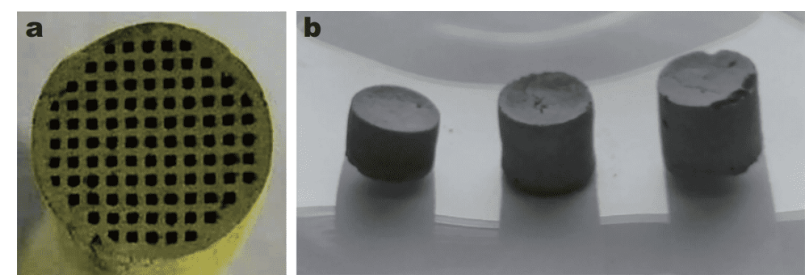

Figure 10 Picture of samples: (a) MIL-101(Cr) monolith, and (b) MIL-101@HIPE composites. Reprinted with permission from Refs [47,101]. Copyright 2015, Elsevier.

$\left(1.08 \mathrm{~g} \mathrm{~g}^{-1}\right.$ for methanol and $1.06 \mathrm{~g} \mathrm{~g}^{-1}$ for water). Regardless, this innovation was an improvement for HIPE after the construction of HKUST-1. In a later report [77], the authors changed the hydrophilic monomer into NIPAM and prepared MIL-100(Cr)@NIPAM (58 wt\% MIL) and MIL-101(Cr)@NIPAM (71 wt\% MIL) monoliths in a similar manner with water sorption values of 0.25 and $0.42 \mathrm{~g} \mathrm{~g}^{-1}$, respectively. The loading of MIL-101(Cr) was up to $92 \%$; however, shrinkage still occurred. In addition, the experimental specific surface area of MIL-101(Cr) $@$ NIPAM is similar to that estimated for MIL-100(Cr) @NIPAM, which may be attributed to the large windows of MIL-101.

\section{Other types of monolithic MOFs}

In addition to the common MOFs summarized above, 
other monolithic MOFs with different metal ions have also been sporadically reported in recent years. For example, Darunte et al. [102] investigated the amine-functionalized $\mathrm{M}_{2}$ (dobpdc) $(\mathrm{M}=\mathrm{Mg}$ and $\mathrm{Mn} ;$ dobpdc $=$ $\operatorname{obpdc}^{4-}: 4,4^{\prime}$-dihydroxy-(1,1'-biphenyl)-3,3'-dicarboxylate) films on the surface of the cordierite monoliths wash-coated with $\alpha$-alumina using $\mathrm{MgO}$ nanoparticles as the metal precursor. The outward orientation of MOF crystals could be achieved in these films, and the monolith products exhibited an outstanding $\mathrm{CO}_{2}$ capture capacity. Rezaei et al. [103] have considerably contributed to the study of Co- and Mg-based MOF monoliths. The immobilization of MOF-74(Ni) and UTSA-16(Co) on cordierite monolith $(600 \mathrm{cpsi})$ was reported, and their adsorption capacities for $\mathrm{CO}_{2}$ capture were investigated. As shown in Fig. 11, using the liquid-phase epitaxy technique, the layer-by-layer (LBL) assembly is focused on the formation of secondary building units followed by a secondary growth procedure. In situ dip coating (ISDC) is employed for the direct growth of the MOF building units. The reliable coating approaches were optimized according to the loading, thickness, and adsorption performance, indicating that the UTSA-16(Co)-cordierite monolith can be successfully obtained only via the ISDC process owing to the high MOF coating (55 wt\%). Both the aforementioned methods can be adopted for the deposition of MOF-74(Ni) on cordierite; however, the combination of LBL assembly and secondary growth was a promising methodology in terms of high MOF coating (52 wt\%). This high loading can be primarily attributed to the pretreatment of the cordierite surface by dentate groups $\left(-\mathrm{OH},-\mathrm{COOH},-\mathrm{NH}_{2}\right)$, resulting in strong adhesion between monoliths and MOF layers as well as in the orientation of nanocrystals in the layers. However, the weight loading of MOFs did not exceed $55 \mathrm{wt} \%$.

Based on a previous study [32], this group presented an improved technique by simplifying the coating procedures and increasing the MOF loadings. Torlon polymer doping (prepared by Torlon in $N$-methyl-2-pyrrolidone (NMP)) was adopted during the preparation of MOF@polymer via the phase separation technique to achieve good adhesion and promote the physical binding of solid surfaces and MOF powders. This was followed by in situ construction of the MOF monolith composite adsorbent (MOF-MCA). Compared with the previous maximum loading, the MOF loading of MOF-74(Ni) and UTSA$16(\mathrm{Co})$ was considerably high, i.e., $73 \mathrm{wt} \%$ and $80 \mathrm{wt} \%$, respectively. Moreover, in both the studies, monolithic MOFs exhibited higher affinity and faster adsorption kinetics when compared with those exhibited by the MOF powders. However, the $\mathrm{CO}_{2}$ uptake was lower, necessitating further research.

Thakkar et al. [104,105] developed the fabrication of 3DP monoliths via the 3D printing technology, facilitating the direct and facile modulation of monolithic materials (e.g., size and geometric shape) through simple programming to satisfy the practical requirements observed in some studies. In accordance with previously conducted studies $[106,107]$, the MOF-74(Ni) or UTSA$16(\mathrm{Co})$ powders were mixed with bentonite clay (as a binder) in ethanol followed by mixing with a PVA solution and $3 \mathrm{D}$ printing in an LBL manner (Fig. 12). The

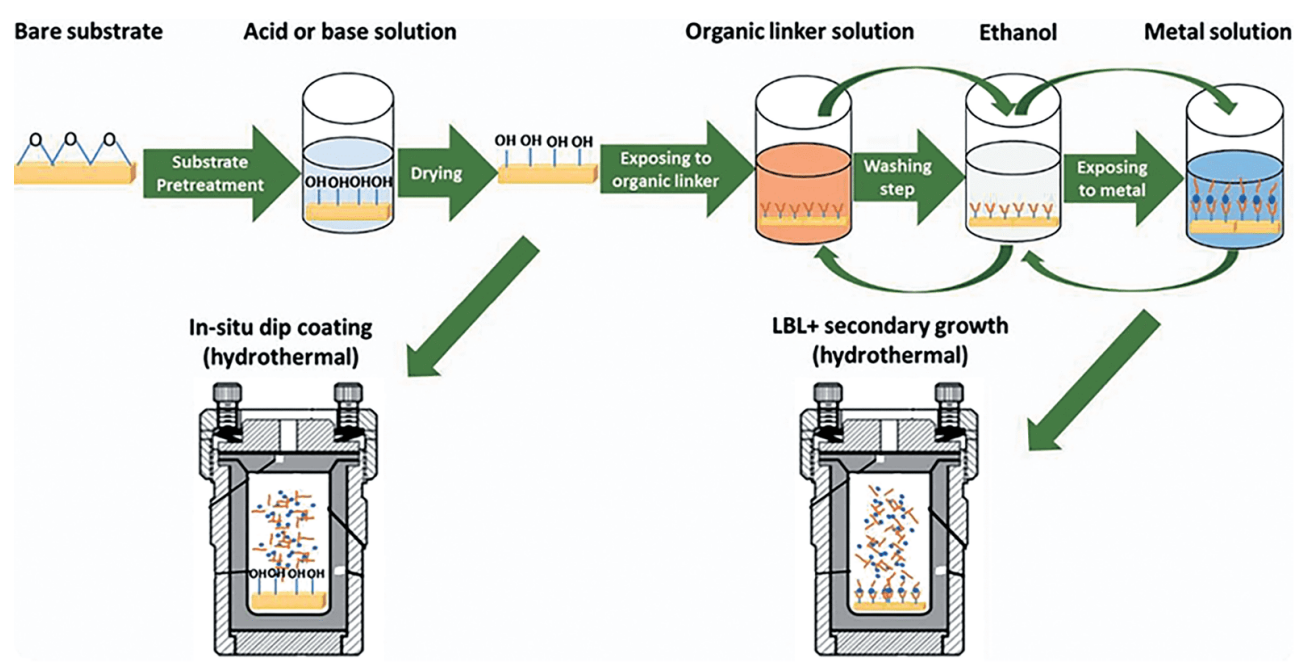

Figure 11 Schematic diagram of preparation of MOF-coated monoliths by LBL assembly + secondary growth and the ISDC techniques. Reprinted with permission from Ref [103]. Copyright 2017, Elsevier. 


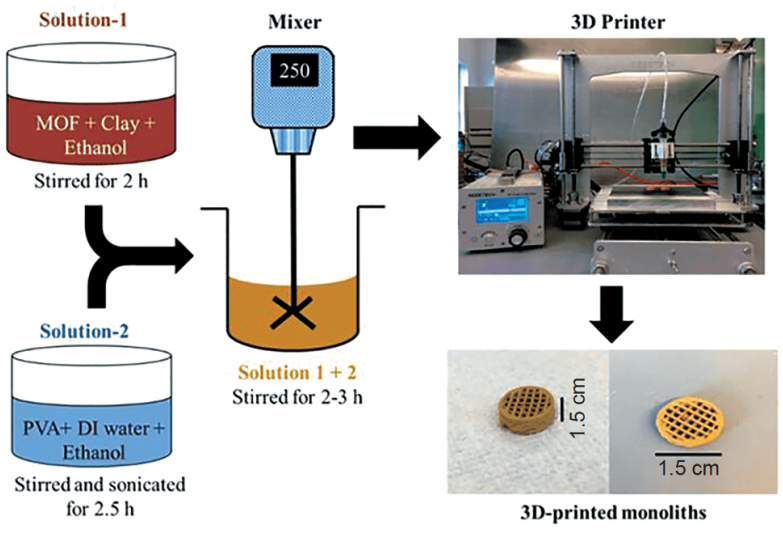

Figure 12 Schematic diagram of the preparation of monolithic MOFs by a 3D printing technique. Reprinted with permission from Ref [29]. Copyright 2017, ACS Publications.

resultant 3D MOF-74(Ni) and UTSA-16(Co) with good mechanical properties showed maximum loadings of 80 $\mathrm{wt} \%$ and $85 \mathrm{wt} \%$, respectively, and fast adsorption kinetics for $\mathrm{CO}_{2}$ capture [29]. Subsequently, they changed the binder to kaolin to avoid disintegration upon exposure to the synthesis liquor and used methyl cellulose (as a plasticizer) and metal impregnation $(\mathrm{Co}, \mathrm{Cu}, \mathrm{Ni}$, or $\mathrm{Cr}$ ) to optimize the MOF loading and structural stability [108]. Co (cobalt oxide inside) was the source of monolithic strength as a $3 \mathrm{D}$ impregnation material because of its inherent properties of high mechanical strength, corrosion resistance, and excellent thermal stability, which have been confirmed previously [109]; these characteristics resulted in a ten-fold enhancement in mechanical strength. This study provides a new proof-of-concept prospect for the economical and efficient industrial application of monolithic MOFs.

\section{CONCLUSIONS AND OUTLOOK}

The shaping of MOF into monolithic state is a necessary route to promote its industrial practicability because of the limitations associated with powdered MOF during industrial engineering (e.g., dustiness, abrasion, clogging, and the pressure drop generated by the packing process). This study aims to summarize the recent advancements associated with monolithic MOFs, including the selection and optimization of the synthesis conditions toward specific MOFs based on different metal ions (e.g., $\mathrm{Cu}, \mathrm{Fe}$, $\mathrm{Zn}, \mathrm{Al}, \mathrm{Zr}$, and Cr-based MOFs), and their excellent performance compared with those of the powdered MOFs. The preparation strategies of MOF monoliths have mainly focused on direct synthesis, the addition of binders, mechanical densification, in situ combination, sol-gel method, and the 3D printing technology. For a specific MOF, it is critical to select and optimize the appropriate route according to the inherent properties and practical application requirements, and create promising candidates for applications in industrial fields, such as for the adsorption of industrial waste gases, removal of heavy metal ions or dyes from sewage, or serving as industrial catalysts or sensors. These requirements encourage the development of monolithic MOF in terms of structure, composition, shape, and performance with respect to the following aspects: low cost, easy handling, non-toxicity/low toxicity, high porosity, high loading capacity, adequate compression resistance, high thermal/ chemical stability, recyclability or regeneration capacity, and excellent adsorption/catalytic/sensing capabilities. Actually, the general objective of ideal MOF monoliths is based on the previous studies on powdered MOFs. Thus, the basic studies that have investigated powdered MOFs cannot be ignored when promoting the industrialization of monolithic MOFs.

Although the shaping studies of monolithic MOFs have made encouraging progress, some challenges remain that are expected to be addressed in the future to realize their engineering applicability. (i) The MOFs in a monolithic state obtained via various approaches are gradually enriched in terms of the structural and functional diversity. However, some well-studied MOFs, such as HKUST-1, ZIF- 8 , and the MIL- $n$ family, dominate the MOF monoliths. Although these MOFs have produced satisfactory results with respect to advanced applications and processability, certain limitations can be observed. This has resulted in the exploration of the possibility of using other MOFs to improve the universality of the current synthesis technologies. (ii) Until now, majority of the studies that have investigated the structural properties of MOF monoliths primarily focus on mechanical stability and high porosity. Less attention has been devoted to thermal and chemical stabilities as well as hydrophobicity, which are practical application requirements. For example, some monolithic MOFs are unstable in water, and irreversible structural decomposition may easily occur after exposure to humid air. In addition, the reduction in the mass transfer rate of the monolithic state has become the biggest limitation in case of industrial applications. This has been alleviated by changing the channel environment (e.g., by introducing a hierarchical network). (iii) Many synthesis strategies have been demonstrated; however, the synthesis mechanism remains unclear, prompting a long and difficult exploration process. (iv) The excellent performance of powdered MOFs is essential 
for the advancement of monolithic MOFs, especially for the promotion of coordination and regulation of the channel environment. In addition to the traditional controlling factors (polarity of solvents, temperature, synthetic time, metal/ligand ratio, and $\mathrm{pH}$ ), other attempts (e.g., addition of templates, modulators, and optimization) along with the drying process have been confirmed to be feasible. However, many concepts related to the formation and application of powdered MOF have not been successfully applied to the field of MOF monolith products, and the functional modification toward monoliths has not been widely studied. (v) Currently, the MOF shaping project is still in the laboratory stage. When being applied in industrial fields, the cost and industrial applicability must not be overlooked with respect to optimization. (vi) The large-scale production of monolithic MOFs is a prerequisite for their applications. However, current synthetic strategies for monolithic MOFs usually require a long reaction time $(>12 \mathrm{~h})$ resulting in a low production rate (usually the space-timeyield (STY) $<300 \mathrm{~kg} \mathrm{~m}^{-3} \mathrm{~d}^{-1}$ ). In conclusion, the preparation of monolithic MOFs still involves many opportunities and challenges. Therefore, further long-term studies must be promoted in this field.

\section{Received 15 September 2020; accepted 2 December 2020; published online 7 February 2021}

1 Ding M, Flaig RW, Jiang HL, et al. Carbon capture and conversion using metal-organic frameworks and MOF-based materials. Chem Soc Rev, 2019, 48: 2783-2828

2 Gonzalez MI, Turkiewicz AB, Darago LE, et al. Confinement of atomically defined metal halide sheets in a metal-organic framework. Nature, 2020, 577: 64-68

3 Jeong YC, Seo JW, Kim JH, et al. Function-regeneration of nonporous hydrolyzed-MOF-derived materials. Nano Res, 2019, 12: 1921-1930

4 Fu JH, Zhong Z, Xie D, et al. SERS-active MIL-100(Fe) sensory array for ultrasensitive and multiplex detection of VOCs. Angew Chem Int Ed, 2020, 59: 20489-20498

5 Lou X, Ning Y, Li C, et al. Bimetallic zeolite imidazolate framework for enhanced lithium storage boosted by the redox participation of nitrogen atoms. Sci China Mater, 2018, 61: 1040-1048

6 Verma G, Kumar S, Vardhan H, et al. A robust SOC-MOF platform exhibiting high gravimetric uptake and volumetric deliverable capacity for on-board methane storage. Nano Res, 2021, 14: 512-517

7 Wang B, Zhang $\mathrm{X}$, Huang $\mathrm{H}$, et al. A microporous aluminumbased metal-organic framework for high methane, hydrogen, and carbon dioxide storage. Nano Res, 2021, 14: 507-511

$8 \mathrm{He} \mathrm{J,} \mathrm{Xu} \mathrm{J,} \mathrm{Yin} \mathrm{J,} \mathrm{et} \mathrm{al.} \mathrm{Recent} \mathrm{advances} \mathrm{in} \mathrm{luminescent} \mathrm{metal-}$ organic frameworks for chemical sensors. Sci China Mater, 2019, 62: $1655-1678$

9 Jiang K, Zhang L, Xia T, et al. A water-stable fcu-MOF material with exposed amino groups for the multi-functional separation of small molecules. Sci China Mater, 2019, 62: 1315-1322

10 Zhang J, Liu F, Gan J, et al. Metal-organic framework film for fluorescence turn-on $\mathrm{H}_{2} \mathrm{~S}$ gas sensing and anti-counterfeiting patterns. Sci China Mater, 2019, 62: 1445-1453

11 Wang R, Liu L, Subhan S, et al. Engineering $\mathrm{pH}$-switchable UiO66 via in-situ amino acid doping for highly selective adsorption of anionic dyes. Chem Eng J, 2020, 395: 124958

12 Iveson SM, Litster JD, Hapgood K, et al. Nucleation, growth and breakage phenomena in agitated wet granulation processes: A review. Powder Tech, 2001, 117: 3-39

13 Shah BB, Kundu T, Zhao D. Mechanical properties of shaped metal-organic frameworks. Top Curr Chem (Z), 2019, 377: 25

14 Gao Z, Wang J, Muhammad Y, et al. Enhanced moistureresistance and excellent photocatalytic performance of synchronous N/Zn-decorated MIL-125(Ti) for vaporous acetaldehyde degradation. Chem Eng J, 2020, 388: 124389

15 Tian T, Zeng Z, Vulpe D, et al. A sol-gel monolithic metalorganic framework with enhanced methane uptake. Nat Mater, 2018, 17: 174-179

16 Duan C, Yu Y, Xiao J, et al. Recent advancements in metalorganic frameworks for green applications. Green Energy Environ, 2020, doi: 10.1016/j.gee.2020.04.006

17 Zhang S, Li JPH, Zhao J, et al. Direct aerobic oxidation of monoalcohol and diols to acetals using tandem Ru@MOF catalysts. Nano Res, 2021, 14: 479-485

18 Rowsell JLC, Yaghi OM. Strategies for hydrogen storage in metalorganic frameworks. Angew Chem Int Ed, 2005, 44: 4670-4679

19 Liu B, Vellingiri K, Jo SH, et al. Recent advances in controlled modification of the size and morphology of metal-organic frameworks. Nano Res, 2018, 11: 4441-4467

20 Nandasiri MI, Jambovane SR, McGrail BP, et al. Adsorption, separation, and catalytic properties of densified metal-organic frameworks. Coord Chem Rev, 2016, 311: 38-52

21 Lorignon F, Gossard A, Carboni M. Hierarchically porous monolithic MOFs: An ongoing challenge for industrial-scale effluent treatment. Chem Eng J, 2020, 393: 124765

22 Yang MH, Duan CX, Zeng XJ, et al. Facile fabrication of nanoscale hierarchical porous zeolitic imidazolate frameworks for enhanced toluene adsorption capacity. Rare Met, 2020, doi: 10.1007/s12598-020-01455-9

23 Küsgens P, Zgaverdea A, Fritz HG, et al. Metal-organic frameworks in monolithic structures. J Am Ceramic Soc, 2010, 93: 2476-2479

24 Tian T, Velazquez-Garcia J, Bennett TD, et al. Mechanically and chemically robust ZIF-8 monoliths with high volumetric adsorption capacity. J Mater Chem A, 2015, 3: 2999-3005

25 Blanita G, Coldea I, Misan I, et al. Hydrogen cryo-adsorption by hexagonal prism monoliths of MIL-101. Int J Hydrogen Energy, 2014, 39: 17040-17046

26 Zhu H, Zhang Q, Zhu S. Assembly of a metal-organic framework into 3D hierarchical porous monoliths using a pickering high internal phase emulsion template. Chem Eur J, 2016, 22: 87518755

27 Wang Z, Song L, Wang Y, et al. Lightweight UiO-66/cellulose aerogels constructed through self-crosslinking strategy for adsorption applications. Chem Eng J, 2019, 371: 138-144

28 Ma X, Lou Y, Chen XB, et al. Multifunctional flexible composite aerogels constructed through in-situ growth of metal-organic framework nanoparticles on bacterial cellulose. Chem Eng J, 2019, 356: 227-235 
29 Thakkar H, Eastman S, Al-Naddaf Q, et al. 3D-printed metalorganic framework monoliths for gas adsorption processes. ACS Appl Mater Interfaces, 2017, 9: 35908-35916

30 Ahmed A, Forster M, Clowes R, et al. Hierarchical porous metalorganic framework monoliths. Chem Commun, 2014, 50: 1431414316

31 Yang C, Liu D, Huang S, et al. Pressure-induced monolithic carbon aerogel from metal-organic framework. Energy Storage Mater, 2020, 28: 393-400

32 Lawson S, Hajari A, Rownaghi AA, et al. MOF immobilization on the surface of polymer-cordierite composite monoliths through in-situ crystal growth. Separ Purif Technol, 2017, 183: 173-180

33 Li W, Henke S, Cheetham AK. Research update: Mechanical properties of metal-organic frameworks-Influence of structure and chemical bonding. APL Mater, 2014, 2: 123902

34 Guo Y, Peng X. Mass transport through metal organic framework membranes. Sci China Mater, 2019, 62: 25-42

35 Zacharia R, Cossement D, Lafi L, et al. Volumetric hydrogen sorption capacity of monoliths prepared by mechanical densification of MOF-177. J Mater Chem, 2010, 20: 2145-2151

36 Coudert FX. Responsive metal-organic frameworks and framework materials: Under pressure, taking the heat, in the spotlight, with friends. Chem Mater, 2015, 27: 1905-1916

37 Finsy V, Ma L, Alaerts L, et al. Separation of $\mathrm{CO}_{2} / \mathrm{CH}_{4}$ mixtures with the MIL-53(Al) metal-organic framework. Microporous Mesoporous Mater, 2009, 120: 221-227

38 Zhu H, Yang X, Cranston ED, et al. Flexible and porous nanocellulose aerogels with high loadings of metal-organic-framework particles for separations applications. Adv Mater, 2016, 28: 76527657

39 Chaudhari AK, Han I, Tan JC. Multifunctional supramolecular hybrid materials constructed from hierarchical self-ordering of in situ generated metal-organic framework (MOF) nanoparticles. Adv Mater, 2015, 27: 4438-4446

40 Mahmood A, Xia W, Mahmood N, et al. Hierarchical heteroaggregation of binary metal-organic gels with tunable porosity and mixed valence metal sites for removal of dyes in water. Sci Rep, 2015, 5: 10556

41 Duan C, Yu Y, Xiao J, et al. Water-based routes for synthesis of metal-organic frameworks: A review. Sci China Mater, 2020, 63: 667-685

42 Piepenbrock MOM, Lloyd GO, Clarke N, et al. Metal- and anionbinding supramolecular gels. Chem Rev, 2010, 110: 1960-2004

43 de Hatten X, Bell N, Yufa N, et al. A dynamic covalent, luminescent metallopolymer that undergoes sol-to-gel transition on temperature rise. J Am Chem Soc, 2011, 133: 3158-3164

44 Duan C, Yu Y, Yang P, et al. Engineering new defects in MIL-100 (Fe) via a mixed-ligand approach to effect enhanced volatile organic compound adsorption capacity. Ind Eng Chem Res, 2020, 59: 774-782

45 Duan C, Yu Y, Hu H. et al. Recent progress on synthesis of ZIF67-based materials and their application to heterogeneous catalysis. Green Energy Environ, 2021 doi: 10.1016/j.gee.2020.12.023

46 Song GQ, Lu YX, Zhang Q, et al. Porous Cu-BTC silica monoliths as efficient heterogeneous catalysts for the selective oxidation of alkylbenzenes. RSC Adv, 2014, 4: 30221-30224

47 Hong WY, Perera SP, Burrows AD. Manufacturing of metalorganic framework monoliths and their application in $\mathrm{CO}_{2}$ adsorption. Microporous Mesoporous Mater, 2015, 214: 149-155

48 Ahmed A, Hasell $\mathrm{T}$, Clowes $\mathrm{R}$, et al. Aligned macroporous monoliths with intrinsic microporosity via a frozen-solventtemplating approach. Chem Commun, 2015, 51: 1717-1720

49 Bradshaw D, Garai A, Huo J. Metal-organic framework growth at functional interfaces: thin films and composites for diverse applications. Chem Soc Rev, 2012, 41: 2344-2381

50 Sindoro M, Yanai N, Jee AY, et al. Colloidal-sized metal-organic frameworks: Synthesis and applications. Acc Chem Res, 2014, 47: 459-469

51 Valizadeh B, Nguyen TN, Stylianou KC. Shape engineering of metal-organic frameworks. Polyhedron, 2018, 145: 1-15

52 Troyano J, Carné-Sánchez A, Avci C, et al. Colloidal metalorganic framework particles: the pioneering case of ZIF-8. Chem Soc Rev, 2019, 48: 5534-5546

53 Song $\mathrm{M}$, Zhao $\mathrm{Y}, \mathrm{Mu} \mathrm{S}$, et al. A stable ZIF-8-coated mesh membrane with micro-/nano architectures produced by a facile fabrication method for high-efficiency oil-water separation. Sci China Mater, 2019, 62: 536-544

54 Hou J, Sapnik AF, Bennett TD. Metal-organic framework gels and monoliths. Chem Sci, 2020, 11: 310-323

55 Duan C, Yu Y, Li F, et al. Ultrafast room-temperature synthesis of hierarchically porous metal-organic frameworks with high space-time yields. CrystEngComm, 2020, 22: 2675-2680

56 DeCoste JB, Peterson GW, Schindler BJ, et al. The effect of water adsorption on the structure of the carboxylate containing metalorganic frameworks Cu-BTC, Mg-MOF-74, and UiO-66. J Mater Chem A, 2013, 1: 11922-11932

57 Duan C, Dong L, Li F, et al. Room-temperature rapid synthesis of two-dimensional metal-organic framework nanosheets with tunable hierarchical porosity for enhanced adsorption desulfurization performance. Ind Eng Chem Res, 2020, 59: 18857-18864

58 Peterson GW, DeCoste JB, Glover TG, et al. Effects of pelletization pressure on the physical and chemical properties of the metal-organic frameworks $\mathrm{Cu}_{3}(\mathrm{BTC})_{2}$ and UiO-66. Microporous Mesoporous Mater, 2013, 179: 48-53

59 Dhainaut J, Avci-Camur C, Troyano J, et al. Systematic study of the impact of MOF densification into tablets on textural and mechanical properties. CrystEngComm, 2017, 19: 4211-4218

60 Sachse A, Ameloot R, Coq B, et al. In situ synthesis of Cu-BTC (HKUST-1) in macro-/mesoporous silica monoliths for continuous flow catalysis. Chem Commun, 2012, 48: 4749-4751

61 Ulker Z, Erucar I, Keskin S, et al. Novel nanostructured composites of silica aerogels with a metal organic framework. Microporous Mesoporous Mater, 2013, 170: 352-358

62 Hassan MH, Haikal RR, Hashem T, et al. Electrically conductive, monolithic metal-organic framework-graphene (MOF@G) composite coatings. ACS Appl Mater Interfaces, 2019, 11: 64426447

63 Qian D, Lei C, Hao GP, et al. Synthesis of hierarchical porous carbon monoliths with incorporated metal-organic frameworks for enhancing volumetric based $\mathrm{CO}_{2}$ capture capability. ACS Appl Mater Interfaces, 2012, 4: 6125-6132

64 Hao GP, Li WC, Qian D, et al. Structurally designed synthesis of mechanically stable poly(benzoxazine-co-resol)-based porous carbon monoliths and their application as high-performance $\mathrm{CO}_{2}$ capture sorbents. J Am Chem Soc, 2011, 133: 11378-11388

65 Moitra N, Fukumoto S, Reboul J, et al. Mechanically stable, hierarchically porous $\mathrm{Cu}_{3}(\mathrm{btc})_{2}$ (HKUST-1) monoliths via direct conversion of copper(ii) hydroxide-based monoliths. Chem Commun, 2015, 51: 3511-3514

66 Truby RL, Lewis JA. Printing soft matter in three dimensions. 
Nature, 2016, 540: 371-378

67 Heinke L, Wöll C. Surface-mounted metal-organic frameworks: Crystalline and porous molecular assemblies for fundamental insights and advanced applications. Adv Mater, 2019, 31: 1806324

68 Heinke L, Gu Z, Wöll C. The surface barrier phenomenon at the loading of metal-organic frameworks. Nat Commun, 2014, 5: 4562

69 Zhao M, Wang Y, Ma Q, et al. Ultrathin 2D metal-organic framework nanosheets. Adv Mater, 2015, 27: 7372-7378

70 Stavila V, Talin AA, Allendorf MD. MOF-based electronic and opto-electronic devices. Chem Soc Rev, 2014, 43: 5994-6010

71 Zhang Z, Müller K, Heidrich S, et al. Light-switchable onedimensional photonic crystals based on MOFs with photomodulatable refractive index. J Phys Chem Lett, 2019, 10: 66266633

72 Gu ZG, Heinke L, Wöll C, et al. Experimental and theoretical investigations of the electronic band structure of metal-organic frameworks of HKUST-1 type. Appl Phys Lett, 2015, 107: 183301

73 Wang Z, Weidler PG, Azucena C, et al. Negative, anisotropic thermal expansion in monolithic thin films of crystalline metalorganic frameworks. Microporous Mesoporous Mater, 2016, 222: 241-246

74 Huo SH, Yan XP. Metal-organic framework MIL-100(Fe) for the adsorption of malachite green from aqueous solution. J Mater Chem, 2012, 22: 7449-7455

75 Yang Q, Zhao Q, Ren SS, et al. Fabrication of core-shell $\mathrm{Fe}_{3} \mathrm{O}_{4} @$ MIL-100(Fe) magnetic microspheres for the removal of $\mathrm{Cr}(\mathrm{vi})$ in aqueous solution. J Solid State Chem, 2016, 244: 25-30

76 Lohe MR, Rose M, Kaskel S. Metal-organic framework (MOF) aerogels with high micro- and macroporosity. Chem Commun, 2009, 40: 6056

77 Wickenheisser M, Paul T, Janiak C. Prospects of monolithic MILMOF@poly(NIPAM)HIPE composites as water sorption materials. Microporous Mesoporous Mater, 2016, 220: 258-269

78 Wang J, Zhu H, Li BG, et al. Interconnected porous monolith prepared via UiO-66 stabilized pickering high internal phase emulsion template. Chem Eur J, 2018, 24: 16426-16431

79 Mahmood A, Zou R, Wang Q, et al. Nanostructured electrode materials derived from metal-organic framework xerogels for high-energy-density asymmetric supercapacitor. ACS Appl Mater Interfaces, 2016, 8: 2148-2157

80 Fairen-Jimenez D, Galvelis R, Torrisi A, et al. Flexibility and swing effect on the adsorption of energy-related gases on ZIF-8: Combined experimental and simulation study. Dalton Trans, 2012, 41: 10752-10762

81 Duan C, Li F, Xiao J, et al. Rapid room-temperature synthesis of hierarchical porous zeolitic imidazolate frameworks with high space-time yield. Sci China Mater, 2017, 60: 1205-1214

82 Guillon O, Gonzalez-Julian J, Dargatz B, et al. Field-assisted sintering technology/spark plasma sintering: Mechanisms, materials, and technology developments. Adv Eng Mater, 2014, 16: 830-849

83 Widmer RN, Lampronti GI, Kunz B, et al. Manufacturing macroporous monoliths of microporous metal-organic frameworks. ACS Appl Nano Mater, 2018, 1: 497-500

84 Mehta JP, Tian T, Zeng Z, et al. Sol-gel synthesis of robust metalorganic frameworks for nanoparticle encapsulation. Adv Funct Mater, 2018, 28: 1705588

85 Xia W, Zhang X, Xu L, et al. Facile and economical synthesis of metal-organic framework MIL-100(Al) gels for high efficiency removal of microcystin-LR. RSC Adv, 2013, 3: 11007-11013

86 Xuan W, Zhu C, Liu Y, et al. Mesoporous metal-organic framework materials. Chem Soc Rev, 2012, 41: 1677-1695

87 Li L, Xiang S, Cao S, et al. A synthetic route to ultralight hierarchically micro/mesoporous $\mathrm{Al}(\mathrm{iii})$-carboxylate metal-organic aerogels. Nat Commun, 2013, 4: 1774

88 Wan W, Zhang R, Ma M, et al. Monolithic aerogel photocatalysts: A review. J Mater Chem A, 2018, 6: 754-775

89 Lirio S, Liu WL, Lin CL, et al. Aluminum based metal-organic framework-polymer monolith in solid-phase microextraction of penicillins in river water and milk samples. J Chromatography A, 2016, 1428: 236-245

90 Lin CL, Lirio S, Chen YT, et al. A novel hybrid metal-organic framework-polymeric monolith for solid-phase microextraction. Chem Eur J, 2014, 20: 3317-3321

91 Hastürk E, Schlüsener C, Quodbach J, et al. Shaping of metalorganic frameworks into mechanically stable monoliths with poly (vinyl alcohol) by phase separation technique. Microporous Mesoporous Mater, 2019, 280: 277-287

92 Qian L, Zhang H. Controlled freezing and freeze drying: A versatile route for porous and micro-/nano-structured materials. J Chem Technol Biotechnol, 2011, 86: 172-184

93 Hastürk E, Höfert SP, Topalli B, et al. Shaping of MOFs via freeze-casting method with hydrophilic polymers and their effect on textural properties. Microporous Mesoporous Mater, 2020, 295: 109907

94 DeCoste JB, Peterson GW, Jasuja H, et al. Stability and degradation mechanisms of metal-organic frameworks containing the $\mathrm{Zr}_{6} \mathrm{O}_{4}(\mathrm{OH})_{4}$ secondary building unit. J Mater Chem A, 2013, 1: $5642-5650$

95 Bambalaza SE, Langmi HW, Mokaya R, et al. Compaction of a zirconium metal-organic framework (UiO-66) for high density hydrogen storage applications. J Mater Chem A, 2018, 6: 2356923577

96 Connolly BM, Aragones-Anglada M, Gandara-Loe J, et al. Tuning porosity in macroscopic monolithic metal-organic frameworks for exceptional natural gas storage. Nat Commun, 2019, 10: 2345

97 Vilela SMF, Salcedo-Abraira P, Micheron L, et al. A robust monolithic metal-organic framework with hierarchical porosity. Chem Commun, 2018, 54: 13088-13091

98 Bueken B, Van Velthoven N, Willhammar T, et al. Gel-based morphological design of zirconium metal-organic frameworks. Chem Sci, 2017, 8: 3939-3948

99 Férey G, Mellot-Draznieks C, Serre C, et al. A chromium terephthalate-based solid with unusually large pore volumes and surface area. Science, 2005, 309: 2040-2042

100 Xiang S, Li L, Zhang J, et al. Porous organic-inorganic hybrid aerogels based on $\mathrm{Cr}^{3+} / \mathrm{Fe}^{3+}$ and rigid bridging carboxylates. J Mater Chem, 2012, 22: 1862-1867

101 Wickenheisser M, Janiak C. Hierarchical embedding of micromesoporous MIL-101(Cr) in macroporous poly(2-hydroxyethyl methacrylate) high internal phase emulsions with monolithic shape for vapor adsorption applications. Microporous Mesoporous Mater, 2015, 204: 242-250

102 Darunte LA, Terada Y, Murdock CR, et al. Monolith-supported amine-functionalized $\mathrm{Mg}_{2}$ (dobpdc) adsorbents for $\mathrm{CO}_{2}$ capture. ACS Appl Mater Interfaces, 2017, 9: 17042-17050

103 Rezaei F, Lawson S, Hosseini H, et al. MOF-74 and UTSA-16 film growth on monolithic structures and their $\mathrm{CO}_{2}$ adsorption performance. Chem Eng J, 2017, 313: 1346-1353 
104

Couck S, Lefevere J, Mullens S, et al. $\mathrm{CO}_{2}, \mathrm{CH}_{4}$ and $\mathrm{N}_{2}$ separation with a 3DFD-printed ZSM-5 monolith. Chem Eng J, 2017, 308: 719-726

105 Li X, Li W, Rezaei F, et al. Catalytic cracking of $n$-hexane for producing light olefins on 3D-printed monoliths of MFI and FAU zeolites. Chem Eng J, 2018, 333: 545-553

106 Thakkar H, Eastman S, Hajari A, et al. 3D-printed zeolite monoliths for $\mathrm{CO}_{2}$ removal from enclosed environments. ACS Appl Mater Interfaces, 2016, 8: 27753-27761

107 Thakkar H, Eastman S, Al-Mamoori A, et al. Formulation of aminosilica adsorbents into 3D-printed monoliths and evaluation of their $\mathrm{CO}_{2}$ capture performance. ACS Appl Mater Interfaces, 2017, 9: 7489-7498

108 Lawson S, Al-Naddaf Q, Krishnamurthy A, et al. UTSA-16 growth within $3 \mathrm{D}$-printed co-kaolin monoliths with high selectivity for $\mathrm{CO}_{2} / \mathrm{CH}_{4}, \mathrm{CO}_{2} / \mathrm{N}_{2}$, and $\mathrm{CO}_{2} / \mathrm{H}_{2}$ separation. ACS Appl Mater Interfaces, 2018, 10: 19076-19086

109 Lee JY, An J, Chua CK. Fundamentals and applications of 3D printing for novel materials. Appl Mater Today, 2017, 7: 120-133

Acknowledgements This work was financially supported by the National Natural Science Foundation of China (22008032 and 22078104), Guangdong Basic and Applied Basic Research Foundation (2019A1515110706), Guangdong Natural Science Foundation (2017A030313052 and 2019A1515011121), the Key Project of Department of Education of Guangdong Province (2016GCZX008), the National Key Research and Development Program (2019YFC1805804), the Innovation Team of Universities in Guangdong Province (2020KCXTD011), the Engineering Research Center of Universities in Guangdong Province (2019GCZX002), the Guangdong Key Laboratory for Hydrogen Energy Technologies (2018B030322005), and the Fundamental Research Funds for the Central Universities.

Author contributions Original idea was conceived by Duan C, Yu Y, Chen D, and Xi H. Manuscript was drafted by Duan C, Yu Y, Li J, Li L, Huang B, Chen D, and Xi H. All authors discussed and commented on the manuscript.

Conflict of interest

The authors declare no competing financial interest.

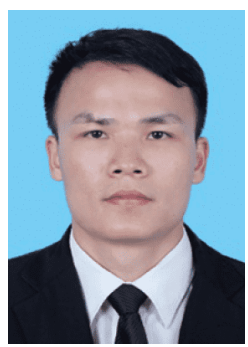

Chongxiong Duan received his $\mathrm{PhD}$ degree in chemical engineering from South China University of Technology in 2019. He is currently an associate professor at the School of Materials Science and Hydrogen Engineering, Foshan University. His research interests focus on the development of porous materials (MOFs, porous carbon, zeolites) and their applications.

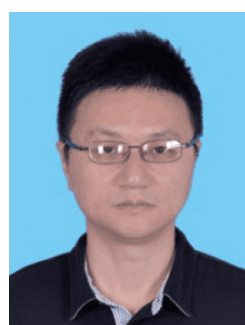

Dongchu Chen received his $\mathrm{PhD}$ degree in material science from Huazhong University of Science and Technology in 2004. He then worked as assistant professor at the School of Science, Foshan University, and currently is a professor at the School of Material Science and Hydrogen Engineering, Foshan University. His research interests focus on the functional materials, applied electrochemistry, and material surface modification.

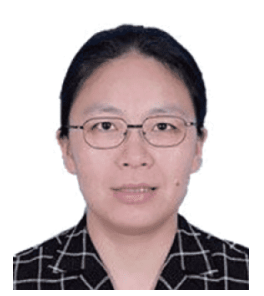

Hongxia Xi received her $\mathrm{PhD}$ degree in chemical engineering from South China University of Technology in 1996. She then worked as a postdoctor for two years at Sun Yat-sen Unviersity, as a visiting scholar for one year at Savoie University, France, and as a senior visiting scholar for six months at The State University of New Jersey, USA. She is currently a professor of chemical engineering at South China University of Technology. Her research interests focus on the development of porous materials and their application.

\section{块状金属-有机骨架材料合成研究进展}

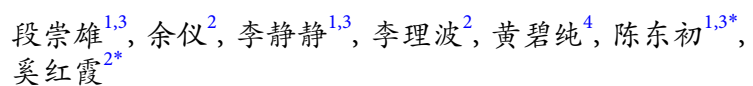

摘要 近年来, 金属-有机骨架材料在制备方面取得了重要进展, 然 而大多数制得的金属-有机骨架材料为分散微晶粉末形态，在工业 应用中易导致粉尘、磨损、堵塞以及压降等一系列问题. 块状金 属-有机骨架材料能克服这些缺陷, 因而表现出了许多优良特性. 本综述中，我们介绍了基于不同金属中心的块状金属-有机骨架材 料的最新进展, 并简要介绍了一些突出例子. 此外, 本文还分析了 块状金属-有机骨架材料在大规模生产和工业应用中面临的挑战和 前景. 